\title{
Mesh Generation Technique for Shock Proof Design of Flat Display Product
}

\author{
JOON-SEONG LEE ${ }^{1, ~ a ~ a n d ~ J I-H O O N ~ Y O O ~} 2, \mathrm{~b}$ \\ ${ }^{1}$ Dept. of Mechanical System Engineering, Kyonggi University, 154-41, Gwanggyosan-ro, \\ Yeongtong-gu, Suwon 16227, Republic of Korea \\ ${ }^{2}$ Undergraduate, Dept. of of Mechanical System Engineering, Kyonggi University \\ ajslee1@kyonggi.ac.kr, bjihun373@naver.coml
}

\begin{abstract}
Keywords: Flat Display, Mesh Generation Algorithm, Fuzzy Technique, Shock Proof, Tetrahedral Element, Hexahedral Element

Abstract. To develop smooth finite element analysis of flat panel display products, we develop an automatic mesh generation system suitable for complex and thin shape objects. In this paper, we propose a new mesh generation technique suitable for flat display products by analyzing existing mesh generation algorithm. We also develop an automatic mesh generation technique that can be linked with knowledge based CAE system based on the proposed algorithm
\end{abstract}

\section{Introduction}

In recent years, CAE has been widely applied in the development of IT-related products such as TFT-LCD, PDP, notebook, and mobile phone, which are representative strategic products, with the finite element method. In product design, CAE plays a vital role in dramatically reducing both the time and cost of new product development by enabling you to review different design alternatives in a short period of time. The finite element method is a process of calculating the respective mechanical relations by subdividing the region of the object to be analyzed into a generally hexahedron or tetrahedron. Therefore, when performing the structural analysis using the finite element method, it is necessary to first divide the object to be analyzed into finite number of elements. The model divided for analysis is called a finite element model or mesh network, and the quality of the mesh representing the shape of the object of interest has a great influence on the structural analysis result. Therefore, a good quality mesh structure[1] is essential to obtain reliable finite element analysis results.

However, as the interest in the design of IT related products has increased recently, flexible curved surfaces have been applied to the design of products, and thin and complicated parts such as mold frames have been used. Therefore, it is acting as a great difficulty. Especially, it is difficult to automate the process of realizing the shape of the IT related product using the hexahedron element which can obtain the reliable structural analysis result, so that much time is required due to the manual operation. In addition, due to the development of computer technology and computer environment, the process of making the analysis network during the actual structural analysis in numerical analysis accounts for about $50 \%$ of the total working time. However, in most of the industrial fields, the mesh generation expert has spent a lot of time and effort creating a good quality network for one model. If the mesh generation process is automated, the finite element analysis time can be shortened, which can shorten the development period of the product and realize concurrent engineering product design [2].

In this paper, a new mesh generation technique is proposed to automate mesh generation process for flat panel display products. For this purpose, we analyze the existing mesh generation algorithm and propose a mesh generation algorithm suitable for a flat and thin flat display product.

\section{Mesh Generation Technique for Flat Panel Display Products}

The research to reduce the generation time of the network and to obtain the good quality network has been actively carried out, and the interest in the automatic network generation program using this is increasing. In the case of tetrahedral elements, it is advantageous that automatic mesh generation is 
possible in almost all geometric shapes, but considering the reliability of analysis results, hexahedral elements[3] are preferred.

In this section, we first applied the existing grid construction method to the curved surface shape to analyze the limit. In addition, the applicability of the tetrahedral element was analyzed by using the tetrahedral element to create the mesh of the flat panel display product. Based on this, a new mesh generation algorithm suitable for the flat display product was suggested by mixing the advantages of the tetrahedral element and the hexahedral element.

\section{Limitations of existing mesh generation}

Generally, when a mesh network is generated by applying a grid configuration method, a quality network can be generated in the interior of the object. However, the quality of the element depends on the basic mesh including the object. In this section, the grid configuration method is applied to the shape of the curved surface where the mesh generation is the most difficult. The first shape is shown in Fig. 1, and the $\mathrm{r} / \mathrm{R}$ of the target shape was changed to $1,0.5$ and 0.25 , and the number of elements of the basic artificial network was varied to 225,625 , and 16,875, respectively. Another application is a dome shape with a slope of $45^{\circ}$ or less and indicated Fig. 2, and the applicability of the tetrahedral element and the influence of the number of elements of the basic element network are analyzed.

The results for the dome column are shown in Fig. 3. As shown in Fig. 3, it is shown that the lattice construction method which implements the shape by determining the coordinate shift of the node and the position information of the element by removing the elements of the boundary part has a limit to realize the curved surface. In the case of using the lattice configuration method, the difference in the shape of the element according to the element density of the elemental mesh is shown in Fig. 4. Even with the same shape, it can be seen that there is a difference in shape implementation according to the element density of the basic mesh.
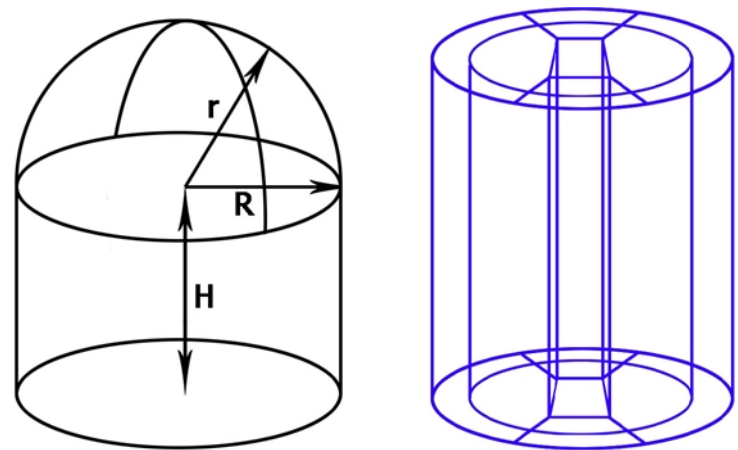

Fig. 1 The cylinder with dome geometry and its CAD configuration

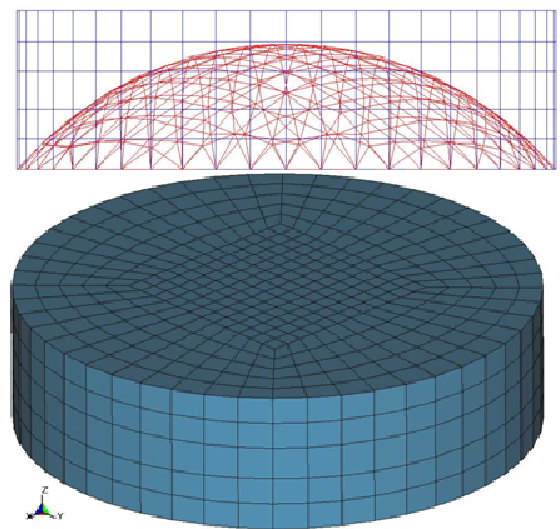

Fig. 2 The skin mesh and based mesh for dome geometry 


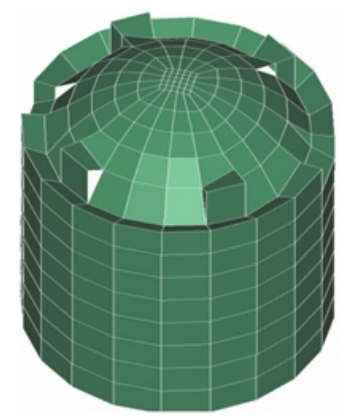

(a) $\mathrm{r} / \mathrm{R}=1.0$

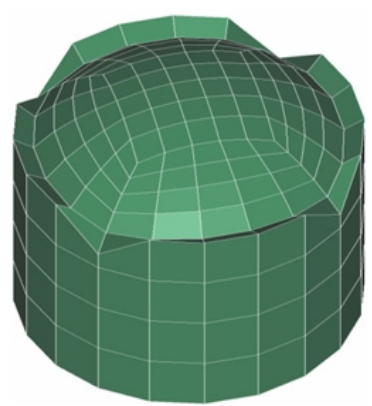

(b) $\mathrm{r} / \mathrm{R}=0.5$

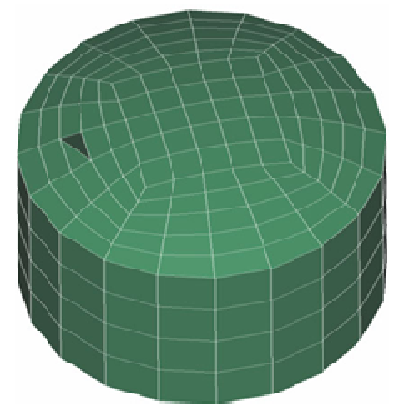

(c) $r / R=0.25$

Fig. 3 Case studies for the sensitivity analysis according to the variation of curvature

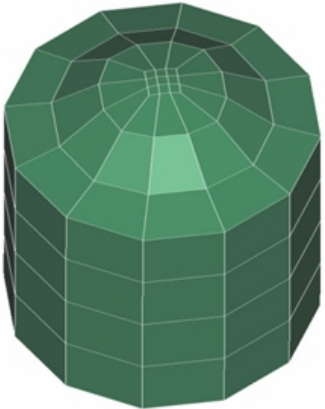

(a) No. of el. $=230$

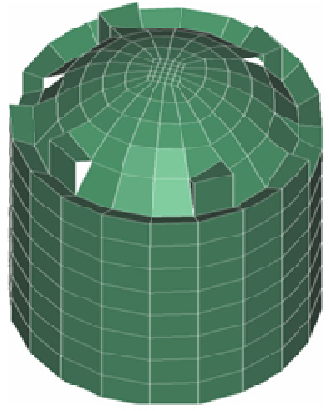

(b) No. of el. $=1500$

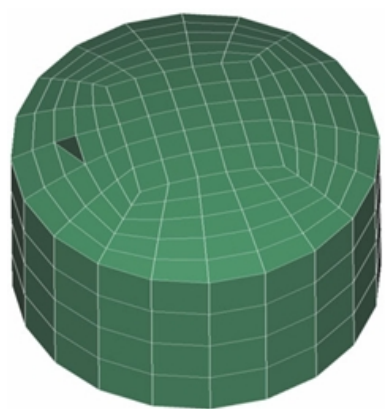

(c) No. of el. $=630$

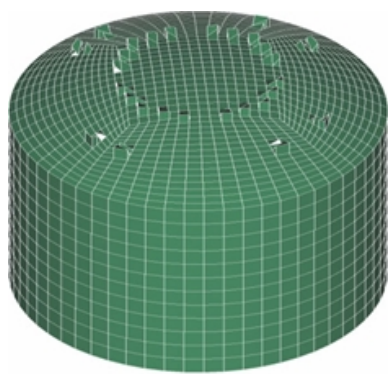

(d) No. of el. $=17,000$

Fig. 4 Case studies for the sensitivity analysis according to the variation of element density

\section{Analysis of tetrahedral mesh for flat panel display products}

In order to analyze the applicability of the mesh network using only the tetrahedral elements with good shape, we applied the impact analysis of the finite element model consisting of the tetrahedron mesh and the finite element model mainly composed of the hexahedron to the mold frame of TFT-LCD TV. Table 1 compares the information of the finite element model created by the mesh generation expert and the information of the finite element model created by the tetrahedral mesh generation module of the commercial CAD program, and the shape is shown in Fig. 5.

Table 1 Information of FE models

\begin{tabular}{c|c|c|c}
\hline \hline \multicolumn{2}{c|}{ FE model } & Manual method & Tetrahedral auto method \\
\hline \multicolumn{2}{c}{ No. of Nodes } & 23,500 & 14,500 \\
\hline \multirow{3}{*}{ No. of Element } & Hexahedron & 13,200 & 0 \\
\cline { 2 - 4 } & Tetrahedron & 0 & 40,500 \\
\cline { 2 - 4 } & Wedge & 180 & 0 \\
\hline \multirow{2}{*}{$\begin{array}{c}\text { The smallest } \\
\text { time step }\end{array}$} & $1^{\text {st }}$ & $1.14 \mathrm{E}-07$ & $7.22 \mathrm{E}-09$ \\
\cline { 2 - 4 } & $2^{\text {nd }}$ & $1.23 \mathrm{E}-07$ & $2.31 \mathrm{E}-08$ \\
\cline { 2 - 4 } & $3^{\text {rd }}$ & $1.26 \mathrm{E}-07$ & $2.31 \mathrm{E}-08$ \\
\hline
\end{tabular}

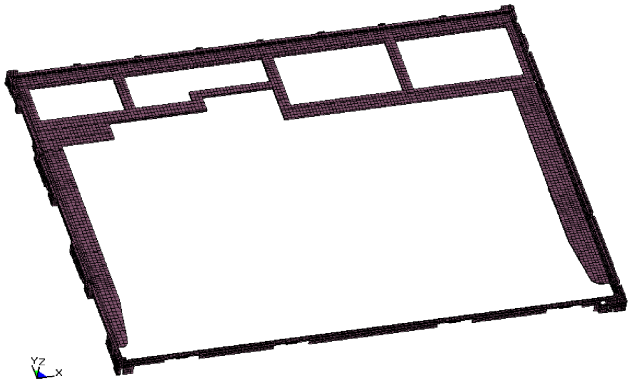

(a) Manual mapped mesh

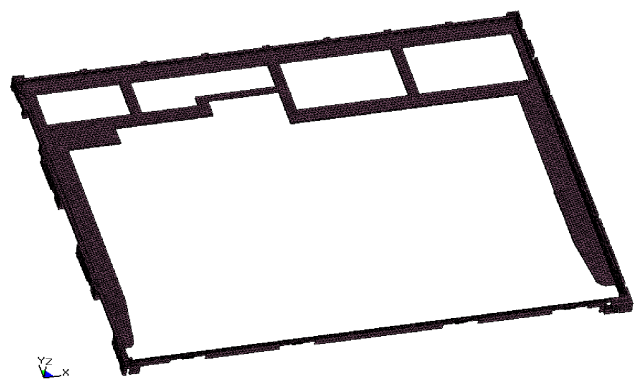

(b) Tetrahedral free mesh

Fig. 5 FE models of Note PC mold frame 


\section{Mesh generation technique based on improved method}

By using the precise position information of the intersection points and the nodes of the basic meshes and the surface meshes, the shape of each basic meshes is changed into the actual shape. Here, the existing method transforms the elements by moving or creating the nodes of the interface, but the new algorithm adds element partitioning method in addition to the movement and creation of the node. That is, among each elementary mesh, the shape of the element is determined by using the intersection information of the elements that meet the surface mesh and the position information of the node, and the element is appropriately divided by referring to the element division knowledge about the shape. Therefore, the newly proposed mesh generation algorithm can generate meshes for various shapes in conjunction with information for shape implementation. The flow diagram of the automatic mesh generation system implemented using the algorithm presented in this chapter is shown in Fig. 6.

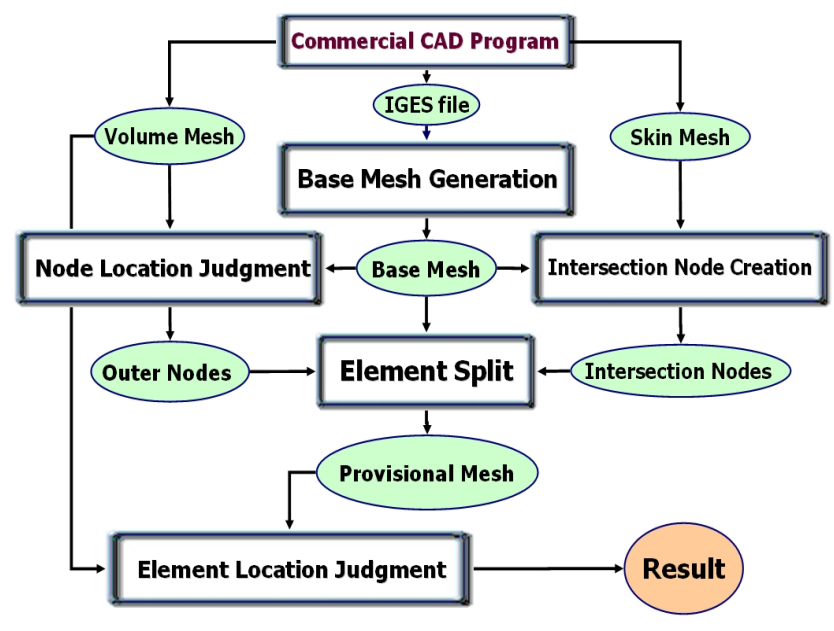

Fig. 6 The proposed mesh generation technique

\section{Conclusions}

A new mesh generation technique suitable for thin and complex flat panel display products is proposed by using the modified grid construction technique. The proposed mesh generation technique is constructed using the advantages of hexahedral and tetrahedral elements. Inside flat panel display products, it is possible to produce good quality hexahedrons that guarantee reliable analysis results, and overcome the limitations of the shape implementation of hexahedrons by applying tetrahedrons to the complex boundaries. . In addition, accurate ring shape information can be determined by using the node and element position information algorithm, and the shape information of each element can be knowledge, and it is possible to generate a mesh for various shapes.

\section{Acknowledgements}

This work was supported by GRRC program of Gyeonggi province. [GRRC 2017-B02, Research on Innovative Intelligent Manufacturing System]

\section{References}

[1] Yagawa, G. and Furukawa, T., Computers and Structures Vol. 47 (2000), p. 1419

[2] Yoon, J.H. and Yang, D.Y., Int. J. of Mechanical Sciences Vol. 30 (2008), p. 887

[3] Ho-Le, K., Computer Aided Design Vol. 20 (2008), p. 27 